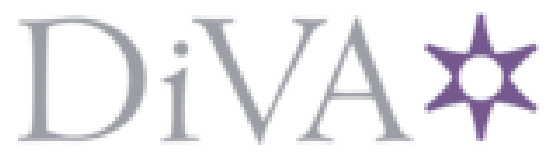

http://www.diva-portal.org

This is the published version of a paper published in Colloids and Surfaces A: Physicochemical and Engineering Aspects.

Citation for the original published paper (version of record):

Khan, A., Redelius, P., Kringos, N. (2016)

Toward a new experimental method for measuring coalescence in bitumen emulsions: A study of two bitumen droplets.

Colloids and Surfaces A: Physicochemical and Engineering Aspects, 494: 228-240

http://dx.doi.org/10.1016/j.colsurfa.2016.01.045

Access to the published version may require subscription.

N.B. When citing this work, cite the original published paper.

Permanent link to this version:

http://urn.kb.se/resolve?urn=urn:nbn:se:kth:diva- 184520 


\title{
Toward a new experimental method for measuring coalescence in bitumen emulsions: A study of two bitumen droplets
}

\author{
Abdullah Khan $^{\mathrm{a}, *}$, Per Redelius ${ }^{\mathrm{b}}$, Nicole Kringos ${ }^{\mathrm{c}}$ \\ ${ }^{a}$ KTH Royal Institute of Technology, SE-10044 Stockholm, Sweden \\ ${ }^{b}$ NYNAS Bitumen AB, SE-149 82 Nynäshamn, Sweden \\ ' KTH Royal Institute of Technology, SE-10044 Stockholm, Sweden
}

\section{H I G H L I G H T S}

- A new experimental method to study coalescence of two bitumen droplets is presented.

- The developed test procedure is a novel state of art, quite simple and repeatable.

- It is possible to study the coalescence process in bitumen emulsions on a large scale.

- This method will serve its widespread applicability in cold mix asphalt technology.

\section{A R T I C L E I N F O}

\section{Article history:}

Received 16 October 2015

Received in revised form 22 January 2016

Accepted 24 January 2016

Available online 5 February 2016

\section{Keywords:}

Bitumen emulsions

Breaking

Coalescence

Relaxation

Sintering

Light transmission
G R A P H I C A L A B S T R A C T

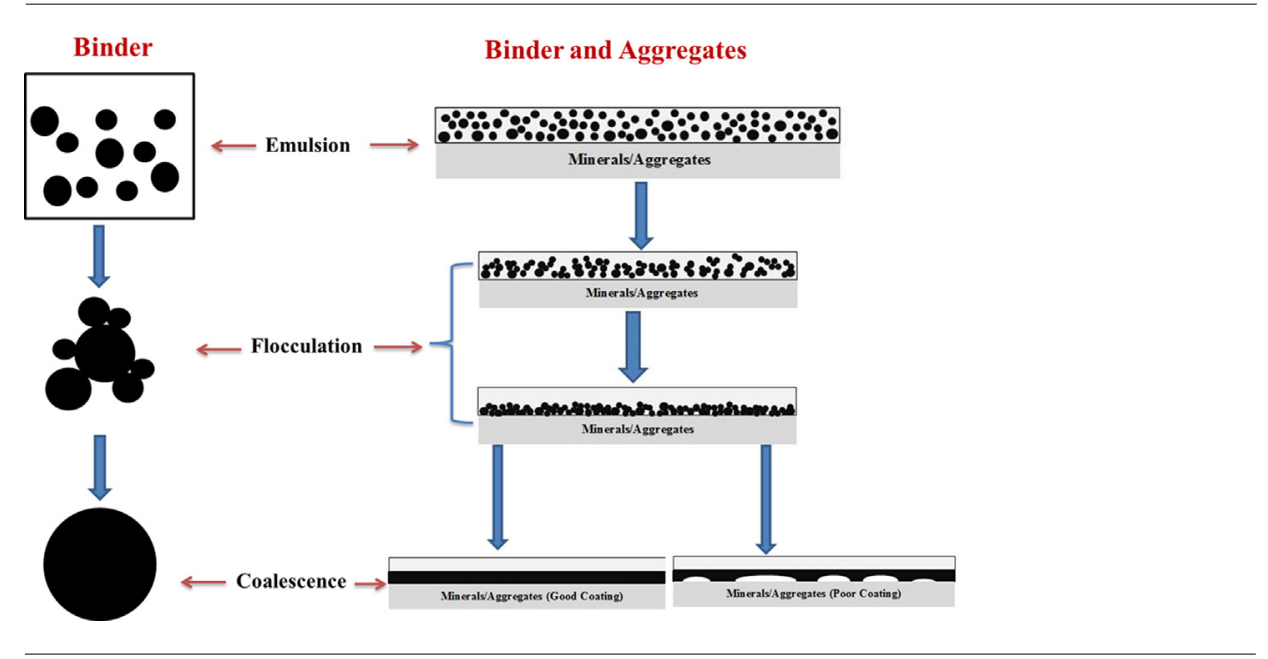

\begin{abstract}
A B S T R A C T
Cold mix asphalt (CMA) emulsion technology could become an attractive alternative for the road industry due to low startup and equipment installation costs, diminished energy consumption and reduced environmental impact. The performance of cold asphalt mixtures produced from emulsions is strongly influenced by a good control of the breaking and coalescence process. The wetting of bitumen on the surface of the aggregates is hereby of major importance for the performance of the asphalt. Premature coalescence of the bitumen emulsions away from the surface, could lead to poor adhesion and decreased mechanical strength of the asphalt. Today, the breaking and coalescence mechanisms of bitumen emulsions are still not fully understood due to their complexities and the lack of fundamental experimental methods and existing models. However, in the past years efforts have been made in defining relationships for understanding the bitumen emulsions. In this paper, a new experimental method is presented to study coalescence of bitumen by using shape relaxation of bitumen droplets in an emulsion environment. The coalescence of spherical droplets of different bitumen have been correlated with neck growth, densification and surface area change during the coalescence process. The test protocol was designed in a controlled climate chamber, to study the coalescence process with varying environmental conditions. The kinetics of the relaxation process was influenced by the temperature as well as other parameters.
\end{abstract}

\footnotetext{
* Corresponding author.

E-mail addresses: abdkha@kth.se, abdullahkhanniazi@gmail.com (A. Khan), per.redelius@telia.com (P. Redelius), kringos@kth.se (N. Kringos).
} 
The research showed that the developed test procedure is repeatable and able to study the coalescence process on a larger scale. However, the relationship between the measured parametric relationships at the larger scale and the bitumen emulsion scale still needs further investigation.

(C) 2016 Elsevier B.V. All rights reserved.

\section{Introduction}

Bitumen is a highly viscous semi-solid at room temperature [1] and is commonly mixed with stone materials for production of asphalt concrete. For a good coating of the stone materials, the viscosity of the bitumen needs to be reduced to enhance its wettability. There are several options to reduce its viscosity to optimum levels, such as heating, dissolving in solvents (cut-back bitumen), foaming the bitumen, or making oil/water type bitumen emulsions. For several reasons, such as its ability to reduce the overall energy usage, cold asphalt mixtures using emulsion technology is an attractive method. Bitumen emulsions are produced by applying high shear force and chemical treatment while warm bitumen and water phase containing emulsifying agents are mixed by the application of mechanical energy to disperse the bitumen into small droplets.

Though the main focus of this paper is bitumen emulsions for the application in asphalt pavements, much can be learned from other emulsion applications. Emulsions, in general, are among the most important colloids in everyday life and have a variety of usage in different fields, such as the paint industry, the food industry, lubrication products, the cosmetic industry and the road construction industry. The application of bitumen emulsions for pavements can be various: e.g. spray applications like surface dressings or chip seals, fog seals, tack and prime coats or mixed with stone material as in slurries or micro surfacing and cold mix paving. Regardless of its application, there are two main parameters controlling the bitumen emulsions: (1) its stability (storage and chemical stability) and (2) its breaking or phase separation upon mixing with aggregates or existing asphalt concrete layers [2]. This paper is focusing on an experimental method towards studying breaking of bitumen emulsions and understanding coalescence mechanism during the phase separation from bitumen emulsions.

For the study of coalescence and flocculation, light scattering or light reflectance and light transmission methods [3-9] have been described in the literature for investigation of crude oil emulsions. These methods include a strong light source and a very sensitive detector to measure light scattered and transmitted. However, the use of conventional light scattering experiments is quite challenging for experiments on dark color crude oil emulsions with multiple scattering events. Nevertheless, some researchers have still tried to apply these techniques on bitumen emulsions and Miller [10] described how to obtain effective scattering parameters from light transmission experiments using a semi-empirical theory for its interpretation. However, much effort is still required to achieve a good resolution at micro scale in real bitumen emulsion environment.

Micromechanical methods like the 'micropipette technique' or the 'vesicle deformation test' were reported in the literature [11-13] for studying the shape relaxation of two droplets. These tests are based on a simple pressurization of the material into spherical shapes. For the coalescence experiments, two distinct pipettes that are coupled with the two different independent micromanipulators are usually brought into contact and pressed against each other to introduce the contact. These types of test methods have shown some promising results, though so far they have been found to be too unpredictable in terms of repeatability to have control over the procedure and, given the large number of variables involved, complex to interpret. These methods do not represent a real bitumen emulsion on a larger scale and drops are forced to coalesce. Moreover, they do not elaborate on the effects of other physio-chemical parameters (e.g. temperature, surfactants, $\mathrm{pH}$ and additives) on breaking and coalescence behavior of bitumen emulsions.

Hence, a reliable and repeatable test procedure for studying the coalescence process in bitumen emulsions under varying conditions is still needed to bring cold asphalt technology to its full market advantage.

\subsection{Breaking E coalescence}

Emulsions can be broken down through various processes such as sedimentation, creaming, Ostwald ripening, flocculation, coalescence or phase inversion. In this study, the main focus is towards flocculation and coalescence as breakdown processes. Flocculation is a reversible process whereby droplets stick together as agglomerates of droplets, without losing their individual integrity. Coalescence is the breaking process of emulsions in which droplets merge together into bigger ones. Bitumen emulsions start breaking or rupturing when in contact with aggregates, as the porous surface of aggregates extracts water away from the emulsion. Furthermore, hydrolysis reactions (releasing of ionic species) take place at the surface of the aggregate minerals. After this, a phase separation by flocculation and coalescence should quickly occur leading to a push out of water from the emulsion as illustrated in Fig. 1a. Two drops of certain radius ' $R$ ' approach each other with negligible initial velocities, touch and form a tiny contact bridge due to Van der Waals interactions. This bridge contact length ' $r_{\mathrm{b}}$ ' quickly expands under the influence of interfacial stress as illustrated in Fig. 1a and finally merges into a larger drop and, as a result, increases the bitumen phase density by minimizing the surface area.

Coalescence is seen as the last step in the breaking process of bitumen emulsions. A continuous film of bituminous binder is developed during coagulation that covers the aggregates followed by curing or setting of the binder, which eventually determines the mechanical strength of the cold asphalt mixture. Good adhesion can be achieved in the case of complete wetting of aggregates by the bitumen and poor adhesion will be achieved if there is a premature coalescence away from the surface or if water is still present at the interface as presented in Fig. 1b.

Studies of coalescing fluid bodies can be traced back to the 19 th century by the work of Reynolds and Lord Rayleigh $[14,15]$. The hydrodynamics of droplet coalescence can be explained in the context of diffusion or capillary forces [16], viscous forces [17-20], inertial forces [21,22] and interfacial forces [22,23]. Especially, droplet coalescence has been studied extensively for systems dealing with microfluidics [24-27] to develop improved coatings in various fields. However, there is still no fundamental test method available today that enables a detailed investigation of the coalescence process for bitumen emulsions. The general practice that has so far been adopted within Europe and the USA for emulsion breaking include the Filler breaking test (EN Standard [28-30]), Resonance Frequency test or Tuning Fork Method (developed in France), Evaporation-Filtration test where coalesced binder 

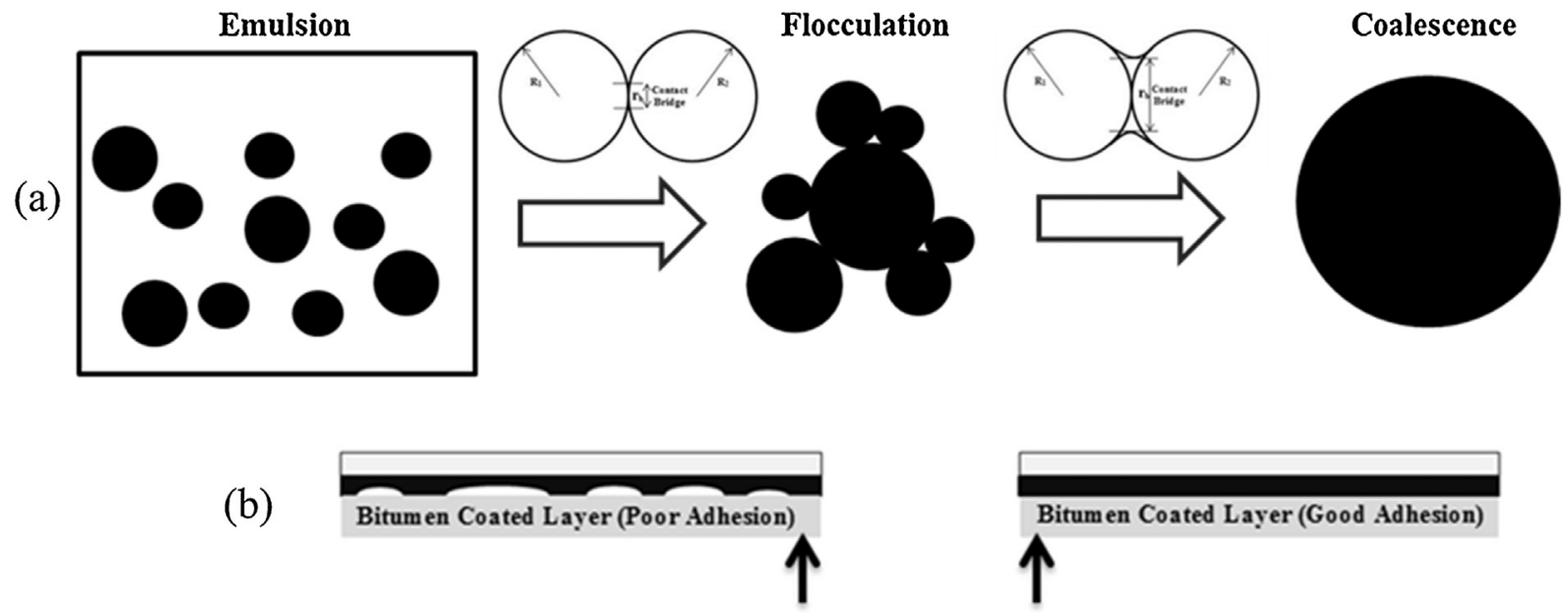

\section{Mineral Substrate}

Fig. 1. (a) Coalescence process in bitumen emulsion, (b) and interaction of bitumen emulsion with aggregates.

is retained on the sieve [31,32], centrifugation, Osmotic pressure, micromanipulation [33], rheology, turbidity and electro-kinetic [34]. Incorporating the practicalities associated with the qualitative comparisons between different emulsions, these tests contribute to an overall characterization of the expected performance but give no fundamental insights which can lead to the prediction or improvement of the material under various circumstances. As such, they can only lead to comparative conclusions but never lead to any real systematic improvement of the design of the emulsions nor enable the optimization for varying storage, field or production conditions.

Several researchers [35-38] have proposed that breaking of bitumen emulsions resembles the classical sintering process known in ceramics, latex paints and aerogels. After adding the breaking additive or destabilizing agent to the emulsion, a gel or network forms which further contracts and becomes a separate phase. According to Frenkel's theory, two oil drops diffuse into each other by a shape relaxation that is mainly driven by surface tension properties [39]. The characteristic time for shape relaxation is thus controlled by a competition between surface tension and viscous dissipation and can be represented as

$\boldsymbol{\tau}_{\text {relaxation }} \propto \frac{\eta \boldsymbol{R}}{\gamma}$

where $\eta$ is the droplet viscosity, $R$ is the characteristic radius of the droplet and $\gamma$ is the droplet surface tension.

It has been observed by Aarts et al. [40] that for viscous driven coalescence $r_{\mathrm{b}}$ grows linearly with $\tau_{\text {relaxation, }}$, whereas in case of coalescence driven by inertial forces, the contact bridge growth relation can be represented as

$r_{\mathrm{b}} \propto \sqrt{\boldsymbol{\tau}}_{\text {relaxation }}$

However, in the case of coalescence of spheres by surface diffusion, there is a very rapid local motion of the bridge in the necking region and $r_{\mathrm{b}}$ exhibits a power law scaling as a function of time which can be written as

$r_{\mathrm{b}}=\left(A \boldsymbol{\tau}_{\text {relaxation }}\right)^{1 / 7},(3)$ where $A$ is a constant related to the material behavior [13].

The majority of emulsion technology problems are related to the challenges of equilibrium between stability and instability of emulsions [41-45] and a better understanding of the mechanisms of stabilization and destabilization of emulsions might lead to enhanced control, thus reducing the risk of their application.

\subsection{Scope and objectives}

The overall goals of the research presented in this paper are to (i) develop a method for the fundamental evaluation of the coalescence process of bitumen emulsions and (ii) enable the investigation of how different physiochemical factors affect the kinetics of the coalescence process. This paper, as part of this effort, focuses on the development of a new experimental procedure that can be conveniently applied to characterize bitumen emulsions and that enables detailed investigation of the effect of its dominant parameters for the coalescence process. In the following, a description of the test set-up is given, followed by a description of the chosen materials for the test demonstration and the test result. At the end of the paper a discussion is given on the validation needs of the test that will enable its practical application.

\section{Methodology}

\subsection{Test set-up}

The method to study coalescence in bitumen emulsions proposed in this study is also based on an optical approach, as mentioned earlier, but its setup is significantly simplified in comparison to the light scattering and light transmission methods. Similar to the micro-pipette and vesicle methods described in the above, the proposed test is based on bringing two droplets in proximity of each other to study their coalescence, but under a more controlled set-up. As shown in Fig. 2. It is very hard to create and handle very small $(\mu \mathrm{m})$ bitumen droplets experimentally, which is in fact one of the largest challenges. In this research, an important assumption is made that by investigating the behavior of bitumen coalescence on a larger scale (order: $\mathrm{mm}$ ) important conclusions can be drawn that are also relevant on the smaller scale (order $\mu \mathrm{m}$ ) in which coalescence actually occurs. This is an assumption that is made to enhance the practicality of the experimental set-up, though needs sufficient validation. Though it is not necessary that there is a 1-1 connection between the two scales, it is important that the investigated parameters at the larger scale have a similar influence on the lower scale.

The basic experimental setup for studying coalescence process in bitumen emulsions is shown in Fig. 3. As it can be seen from Fig. 3a, the set-up consists of a camera, a light source and a climate chamber with optical transparent walls. In addition to these, the test setup contains a transparent glass container, two L-shaped 


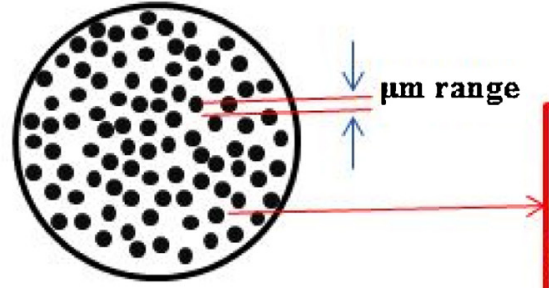

(a). Emulsion drop

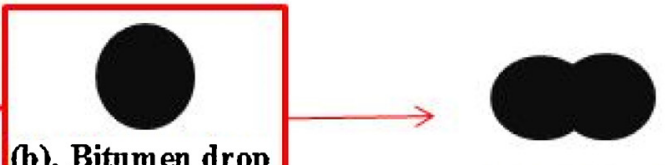

(b). Bitumen drop (mm range)

(c). Coalescence

Fig. 2. Up-scaling assumption for bitumen droplet size in coalescence experiments.

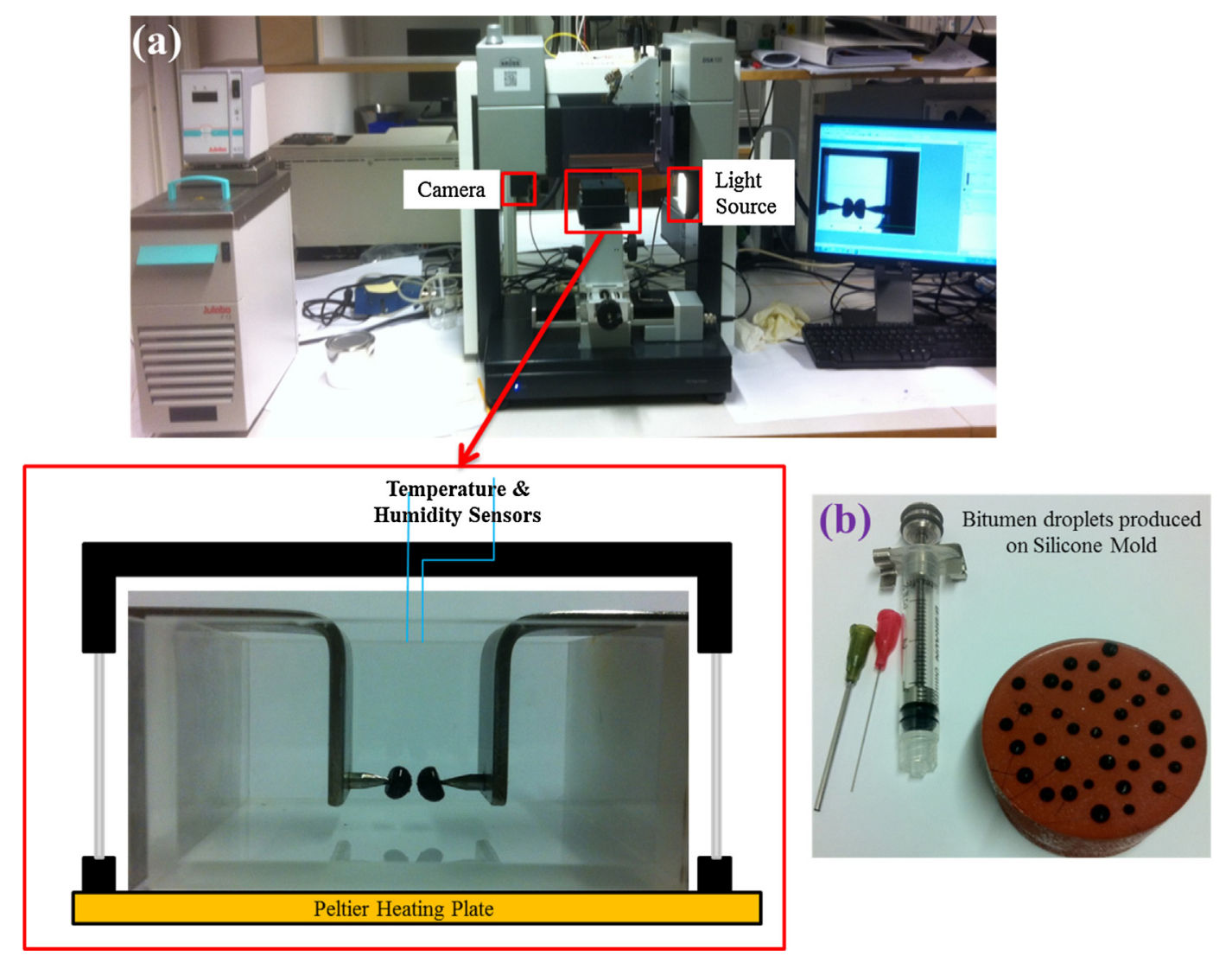

Fig. 3. (a) Experimental setup for studying coalescence process of bitumen droplets, (b) and formation of bitumen droplet on Silicone Mold.

probes hooked with a vertical support of the glass container, temperature and humidity sensors and a Peltier heating panel that is connected to the temperature bath. Bitumen drops of 1-4 mm size are attached with L-shaped probes and brought into contact with each other for the coalescence to happen. The transparent glass container is filled with the relevant solvents (water phase with and without additives).

The temperature of the experiment can be easily controlled and, in this set-up, can be varied from $-30^{\circ} \mathrm{C}$ up to little below the boiling point of water. The setup was also calibrated for the temperature and humidity before measurements. With the help of a camera having a pixel resolution of $780 \times 580 \mathrm{px}$ with a frame rate of $25 \mathrm{fps}$, the kinetics of the coalescence process can be followed and images are recorded at regular intervals.

\subsection{Coalescence experiment}

Bitumen samples are heated in the oven to form a flowing liquid (160-180 ${ }^{\circ} \mathrm{C}$ depending on bitumen grade) and then with the help of different sizes of the syringe needles, droplets of different diam- eters are produced at the surface of silicon rubber as it has nonstick surface for bitumen (presented in Fig. 3b). After this, rubber surface containing bitumen droplets is placed in a refrigerator at for 2-3 $\mathrm{h}$. Bitumen drops are stabilized at $-20^{\circ} \mathrm{C}$, in order to maintain their shape as droplets. But coalescence experiments were carried out at higher temperatures and coalescence experiments was started when an individual drop reached the test temperature.

The two bitumen droplets are brought in contact with each other to analyze coalescence in different environments. In the vertical experimental setup, droplets come into contact due to gravity but in the case of horizontal configuration, a very small force is applied to bring two drops in contact and then leave them to relax naturally. If the coalescence test is performed above ambient temperature, then the temperature of the chamber is first set to the required temperature, which needed some time to stabilize. After temperature stabilization, the small droplets are attached with the help of a sharp edge tweezer to the fine ends of L-shaped probes (as shown in Fig. 3). After this, these probes are placed in a glass container in the climate chamber, which is filled with water and additives to simulate the emulsion environment. When these probes are 


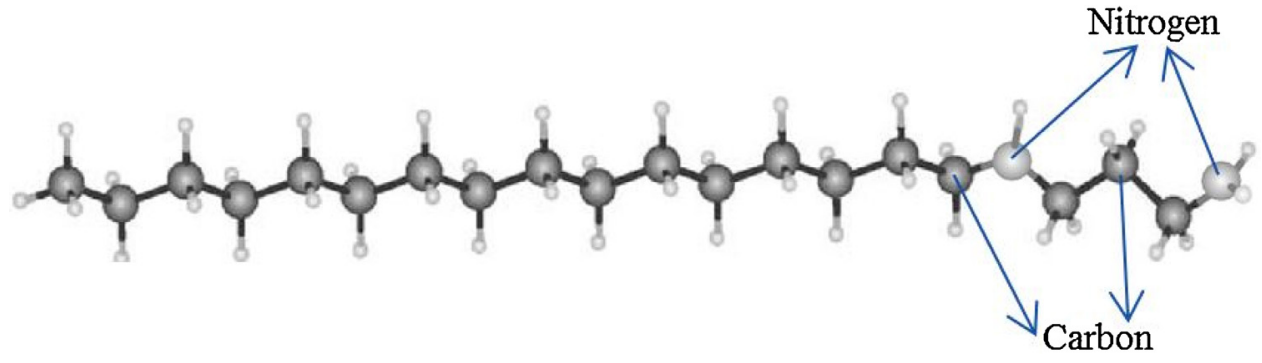

Fig. 4. $N$-Hexadecanoic-1,3-propanediamine. (Black is carbon, light gray is nitrogen atoms).
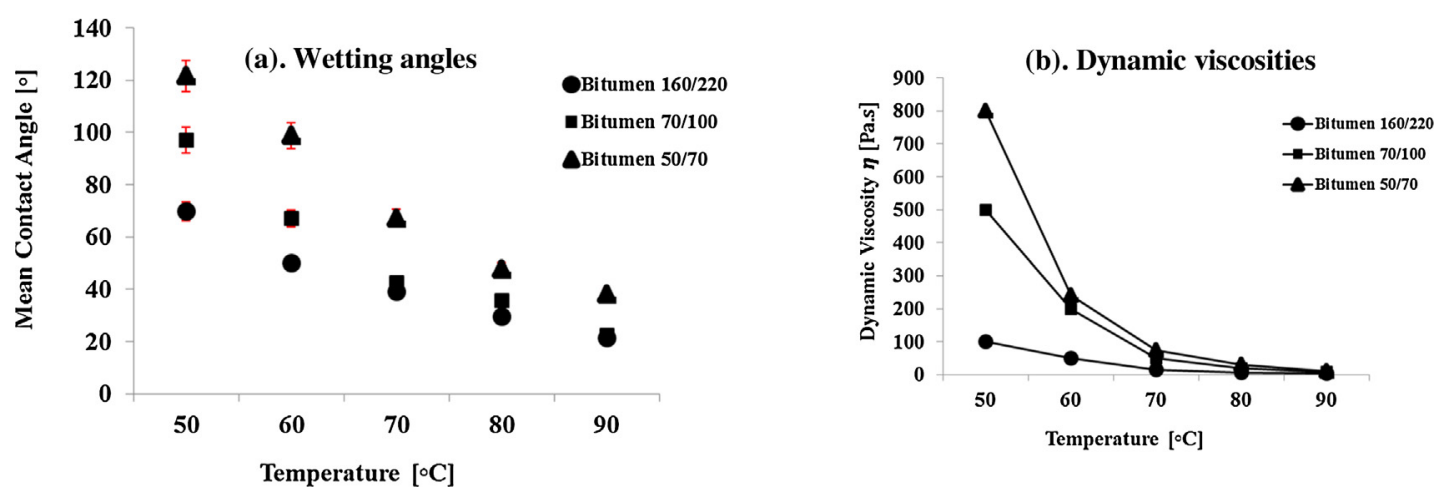

Fig. 5. (a) Contact/wetting angles at different temperatures, (b) dynamic viscosities of three bitumen grades at different temperature.

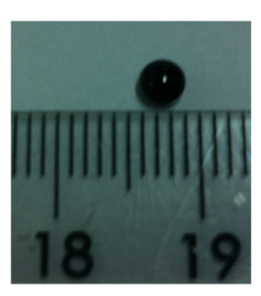

Time (mins)

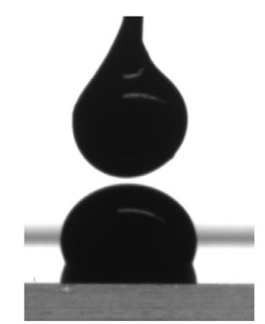

$\mathrm{t}=0 \mathrm{mins}$

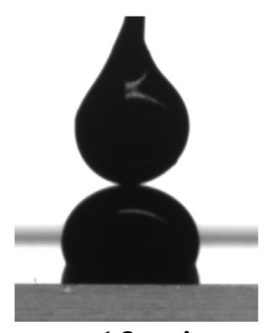

$\mathrm{t}=13 \operatorname{mins}$

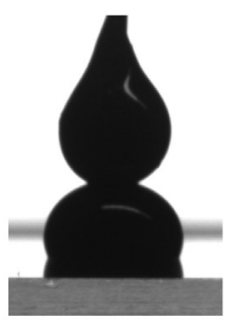

$\mathrm{t}=30$ mins

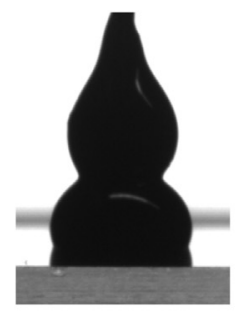

$\mathrm{t}=45$ mins

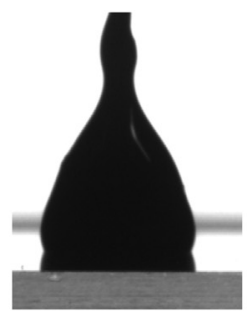

$\mathrm{t}=98$ mins

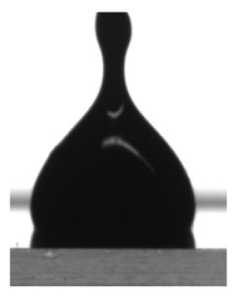

$\mathrm{t}=157$ mins

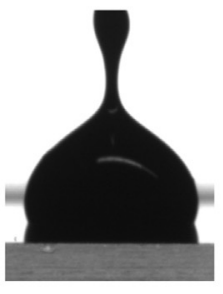

$\mathrm{t}=211$ mins

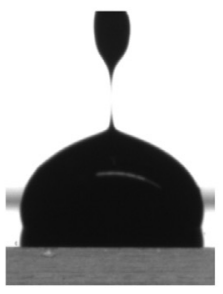

$\mathrm{t}=240 \operatorname{mins}$

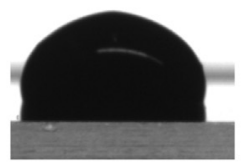

$\mathrm{t}=252$ mins

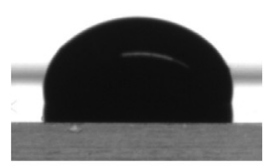

$\mathrm{t}=290$ mins

$\mathrm{t}=1440$ mins

Fig. 6. Coalescence of bitumen $160 / 220$ in air at $22.5^{\circ} \mathrm{C}$.

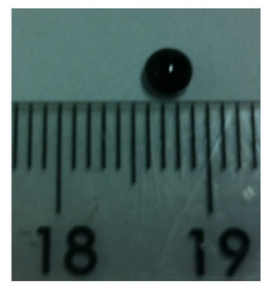

Time (mins)

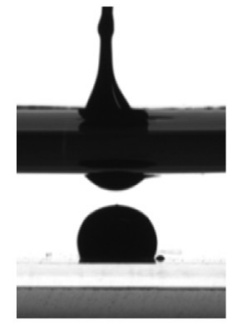

$\mathrm{t}=0 \mathrm{mins}$

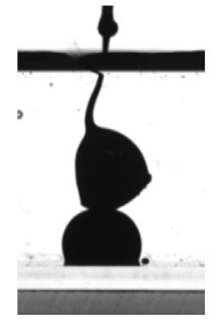

$\mathrm{t}=9$ mins
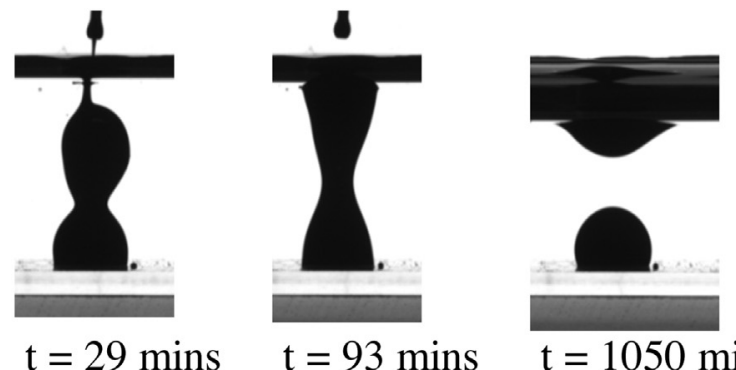

$\mathrm{t}=1050 \mathrm{mins}$

Fig. 7. Coalescence of bitumen $160 / 220$ in water at $22.8^{\circ} \mathrm{C}$. 
placed in a water phase container, the drops are far away from each other initially. These two drops are brought together manually in a way that they are almost touching each other but in reality these drops are separated by a very small distance (approximately by order half $\mu \mathrm{m}$ ). Hence, there is no applied force which is influencing the droplet deformation and relaxation process. Recordings are made of the process of droplets that start to coalesce when brought together in contact. From this, the coalescence times are analyzed that may vary for different binder grades, droplet sizes, test temperatures, types of emulsifiers, additives (salts and salt concentrations, adhesion promoters etc.), variation in $\mathrm{pH}$ and different solvents.

\subsection{Materials}

In this study, an emulsion grade of unaged and unmodified straight run Nynas bitumen with penetration grades 160/220, 70/100 and 50/70 were used to demonstrate the feasibility, efficiency and applicability of the new proposed experimental method. The basic physical properties and chemical constituents according to latroscan MK 6S chromatography analysis and the SARA (Saturates, Aromatics, Resins and Asphaltenes) fractions for three grades of Nynas bitumen are shown in Tables 1 and 2 respectively.

To simulate the emulsion environment, Redicote EM $44^{\circledR}$ from AkzoNobel was added to the water phase in the experiment by $1.0 \mathrm{wt} \%$. Redicote EM44 is basically an amine based ( $N$-tallow alkyltrimethylene-diamine as presented in Fig. 4, but containing additional functional groups) standard emulsifier for rapid and medium setting cationic bitumen emulsions which is being applied in Europe, Asia Pacific and Africa for cationic rapid set (CRS), opengraded cold mixes, penetration macadam, surface dressing or chip seal, and tack coat or bond coat applications. EM44 has a viscosity and density around $450 \mathrm{mPa}$ s and $930 \mathrm{~kg} / \mathrm{m}^{3}$ at $20^{\circ} \mathrm{C}$ respectively, with yellow to red liquid appearance. It is soluble in alcohol or hydrocarbon solvent but only dispersible or insoluble in water. Redicote EM44 is a commercial emulsifier for bitumen so the exact chemical composition is not given. However, most of commercial emulsifiers consist of mixture of more than one emulsifier to give the optimal properties for a particular application [2].

Developing a method to study coalescence process in bitumen emulsion involved a lot of brainstorming. The first challenging task was how to produce bitumen droplets of different sizes and how to handle them, but that was achieved simply by different trials and efforts. After successful formation of bitumen droplets, the frozen stored droplets were brought into vertical contact using very fine tweezer by placing one drop at the glass substrate and the other drop was attached to the vertical needle just on the top of first drop. Firstly, the two drops were let to relax on top of each other, in air and at ambient temperature under the influence of gravity. This simple test was performed just to know whether the relaxation experiment using this setup will work or not. The first relaxation experiment was quite successful in air. After this, several tests were performed with different viscosity grades to evaluate the difference in relaxation times. Later on the environment was replaced with distilled water which is more close to real emulsion systems but the vertical suspended bitumen drop faced Buoyancy force, which is an upward force exerted by a fluid that opposes the weight of an immersed object. This problem was overcome by a new horizontal set up design which will be elaborated in detail later in the text.

Different combinations of water phases with and without additives were prepared to analyze relaxation process of bitumen droplets in the actual emulsion environment using the new method. The coalescence of two bitumen drops were studied in pure water, water with emulsifier, salt (Reagent Grade Anhydrous $\mathrm{CaCl}_{2}$ from Sigma-Aldrich), and certain level of $\mathrm{pH}$ (hydrochloric acid). In addition to that, water phase was mixed with other solvents like Methanol (Reagent grade, VWR), Ethanol (SOLVECO
Analytical grade, 99.5\%), 2-propanol (reagent grade, VWR), Glycerol (Sigma-Aldrich, Reagent Plus 99\%). The ratio for each solvent mixture (e.g. $90 \%$ water phase $+10 \%$ organic solvent).

\section{Experimental results}

\subsection{Contact angle measurements}

Being a visco-elastic material, the characteristic relaxation time of the bitumen is strongly dependent on the physical and chemical properties of the bitumen. For this reason, the wettability profiles of three bitumen grades were analyzed at different temperatures with contact angle methods using KRÜSS DSA100 equipment as shown in Table 3 and Fig. 5. From this, the enhanced wettability at high temperature and with softer grades of the binder can be clearly seen.

In Fig. 5, the measured mean contact angles are plotted for the three bitumen grades at different temperatures. All the contact angle measurements were performed after drop deposition and contact angle were recorded at $3 \mathrm{~min}$ of deposition time frame, It should be noted that this timeframe does not ensure that equilibrium is reached. Equilibrium time scales will be different for each binder at different temperatures. From the behavior of each bitumen grade it can be seen that the wettability behavior with respect to temperature, follow similar trend to that of viscosity as presented in Fig. 5a and b, showing that wetting or relaxation spreading is directly related to the viscosity of the bitumen at different temperatures. The viscosity data is a converted data from logarithmic scale (found in the literature for three different grades of same base binder) to a linear scale. The kinetics of shape relaxation of bitumen droplets can be related to the different physio-chemical parameters such as viscosity and surface energy of the bitumen as well as droplet size (see Eq. (1)). Moreover, surface free energies of both bitumen and the substrate are also equally important for the wettability (coating and adhesion) as well as relaxation process (coalescence or cohesion). The ratios between surface energy and viscosity $(\Upsilon / \eta)$ of each binder can be used to predict the rate of relaxation or an estimate for the average relaxation time [37].

\subsection{Coalescence of bitumen drops in air}

To observe the relaxation times at ambient temperature, the coalescence experiments were initially performed in air using a vertical contact approach where two bitumen droplets were brought into contact by force of gravity. In Fig. 6, an example of the test done with the softer grade binder 160/220 in an air environment (i.e. no moisture was present) is presented. In this experiment, the air humidity was $45.5 \%$ and the temperature was $22.5^{\circ} \mathrm{C}$. It almost took $24 \mathrm{~h}$ to coalesce for softer grade binder 160/220 at ambient temperature as shown in Fig. 6.

\subsection{Coalescence of bitumen drops in water}

To re-create the climate in which a bitumen droplet in an emulsion finds itself, in the second stage, the coalescence of the droplets was investigated in an environment with water. Fig. 7 shows the coalescence in water of the same bitumen presented in Fig. 6. In this, the test temperature was $22.8^{\circ} \mathrm{C}$. As it can be seen from Fig. 7, the Buoyancy effects were dominant and made it difficult for the unmodified bitumen to complete its coalescence.

Despite several adjustments to this under water set-up, including temperature changes and solvents with lower densities than water, this vertical set-up was not found to be reliable in its current design and a horizontal approach was developed. In Section 3.4 
Table 1

Properties of three different grades of base bitumen.

\begin{tabular}{|c|c|c|c|c|c|c|c|}
\hline \multirow[t]{2}{*}{ Bitumen grade } & \multicolumn{2}{|c|}{ Penetration $(\mathrm{mm} / 10)$} & \multicolumn{2}{|c|}{ Softening point $\left({ }^{\circ} \mathrm{C}\right)$} & \multirow{2}{*}{$\begin{array}{l}\text { Dyn. viscosity } \\
\text { (Pas)@60 } 90\end{array}$} & \multirow{2}{*}{$\begin{array}{l}\text { Kin. viscosity }\left(\mathrm{mm}^{2} / \mathrm{s}\right) \\
\text { @ } 135^{\circ} \mathrm{C}\end{array}$} & \multirow{2}{*}{$\begin{array}{l}\text { Fraass breaking } \\
\text { point }\left({ }^{\circ} \mathrm{C}\right)\end{array}$} \\
\hline & Min & $\operatorname{Max}$ & Min & Max & & & \\
\hline $50 / 70$ & 50 & 70 & 46 & 54 & 145 & 295 & -8 \\
\hline $70 / 100$ & 70 & 100 & 43 & 51 & 90 & 230 & -10 \\
\hline $160 / 220$ & 160 & 220 & 35 & 43 & 30 & 135 & -15 \\
\hline
\end{tabular}

Table 2

Generic fractions of iatroscan analysis (SARA fractions).

\begin{tabular}{|c|c|c|c|c|}
\hline Bitumen grade & Saturates (\%wt.) & Aromatics (\%wt.) & Resins (\%wt.) & Asphaltenes (\%wt.) \\
\hline $50 / 70$ & 3.6 & 52.4 & 22.7 & 21.3 \\
\hline $70 / 100$ & 6.4 & 48.6 & 26.2 & 18.8 \\
\hline $160 / 220$ & 9.0 & 54.3 & 22.2 & 14.5 \\
\hline
\end{tabular}

Table 3

Wettability of three different grades of base bitumen.

\begin{tabular}{|c|c|c|c|c|c|}
\hline \multirow[t]{2}{*}{ Bitumen grade } & \multicolumn{5}{|c|}{ Wettability profiles of bitumen drops at glass substrates } \\
\hline & $50^{\circ} \mathrm{C}$ & $60^{\circ} \mathrm{C}$ & $70^{\circ} \mathrm{C}$ & $80^{\circ} \mathrm{C}$ & $90^{\circ} \mathrm{C}$ \\
\hline $50 / 70$ & & & & & \\
\hline $70 / 100$ & & & & & \\
\hline $160 / 220$ & & & & & \\
\hline
\end{tabular}

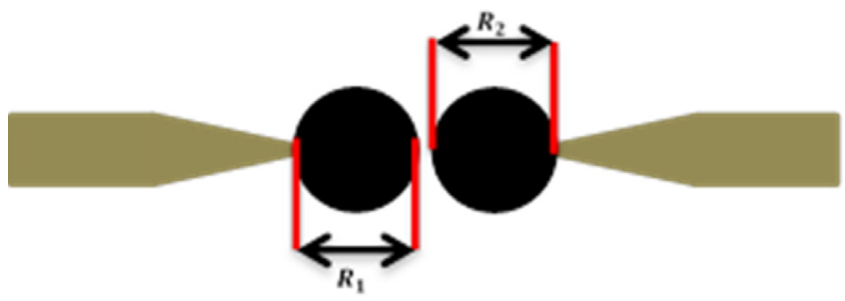

Fig. 8. New Setup for drop coalescence.

more discussion on the possible modification of the vertical set-up is given.

\subsection{Coalescence of bitumen drops in water (new setup design)}

As shown in Fig. 8, a horizontal drop approaching scheme was selected instead of vertical approach to overcome Buoyancy effects in water environment. In this configuration, both droplets have now the same level of immersion potential.

In Fig. 9, the coalescence process of bitumen 160/220 is shown in this new test configuration. The temperature of the water was $21.8^{\circ} \mathrm{C}$. The relaxation time measured was similar (approximately $24 \mathrm{~h}$ ) to that measured in air for the same binder.

To investigate the influence of accelerating the relaxation kinetics, the water temperature was raised to $50^{\circ} \mathrm{C}$. Representative images for the three bitumen penetration grades $160 / 220,70 / 100$, and 50/70 are shown in Fig. 10. The early stage of coalescence represents neck formation and it was very rapid as compared to the last stages of the coalescence process.
The relaxation times $\tau_{\text {relaxation, of three bitumen grades were }}$ also studied as function of droplet sizes and temperatures of the water phase as plotted in Fig. 11(a-c). All experiments were repeated 2-3 times to ensure that the representative behavior was covered. The results from three droplet size ranges, e.g. small size (1-2 mm), medium size (2-3 mm) and large size (3-4 mm) are plotted for all three binder grades. It can be noticed from the graphs that at high temperatures and softer binder grades, the relaxation times of the different binders do not vary much with respect to droplet size. However, at lower temperatures and harder bitumen grades, influence of droplet size on $\tau_{\text {relaxation }}$ is very dominant. It can also be observed that coalescence time is prolonged with large size droplets, which is according to the relation as presented in Eq. (1).

According to Frenkel's theory of viscous sintering, the time of relaxation is directly proportional to the ratio between viscosity and surface tension of each bitumen grade which can be observed here as well. The coalescence time or relaxation times at $50^{\circ} \mathrm{C}$ for binders $160 / 220,70 / 100$, and 50/70 were 7,13 and 20 min respectively. The droplet shrinkage is very rapid in the early stages of coalescence and bridge contact ' $r$ ' ' grows linear with time for all three binders, which also agrees with the Finite Element based theoretical calculations of Martinez-Herera and Derby [20]. Moreover, if ratios between surface energy and viscosity $(\Upsilon / \eta)$ of each binder are compared, soft binder will have higher values as compared to the harder binder grade, which explains the extremely fast and extremely low relaxation process respectively [37]. So far, our method has been tested for the same penetration grade binder but from three different (chemical) origins. The results have shown that the method was sensitive enough to characterize different binders available in the market [46]. 

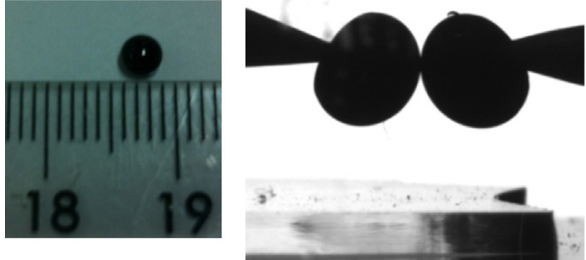

$\mathrm{t}=1 \mathrm{mins}$

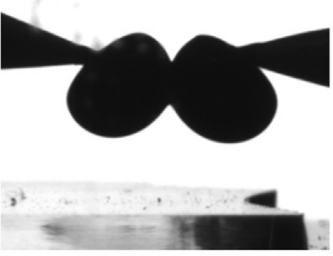

$\mathrm{t}=72 \operatorname{mins}$

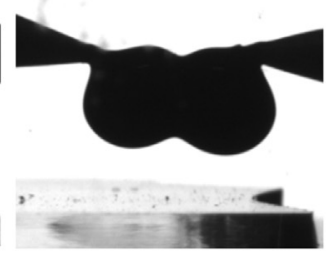

$\mathrm{t}=452$ mins

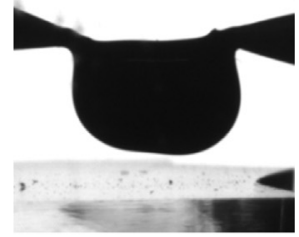

$\mathrm{t}=1464 \operatorname{mins}$

Fig. 9. Coalescence of bitumen $160 / 220$ in water at $22.8^{\circ} \mathrm{C}$.

\section{Binder 160/220 [water, Temperature $50{ }^{\circ} \mathrm{C}$ ]}

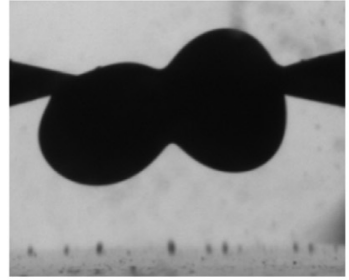

$\mathrm{t}=1 \mathrm{mins}$

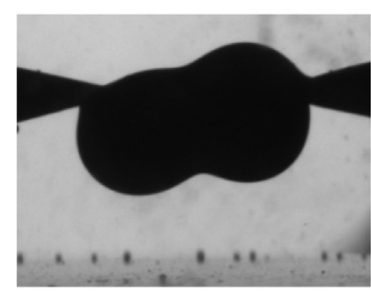

$\mathrm{t}=2 \operatorname{mins}$

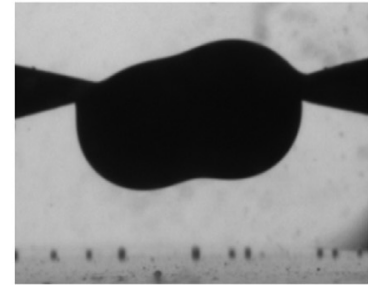

$\mathrm{t}=3 \mathrm{mins}$

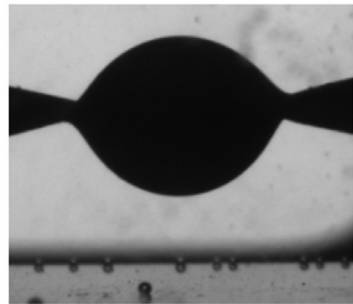

$\mathrm{t}=7$ mins

Binder 70/100 [water, Temperature $50^{\circ} \mathrm{C}$ ]

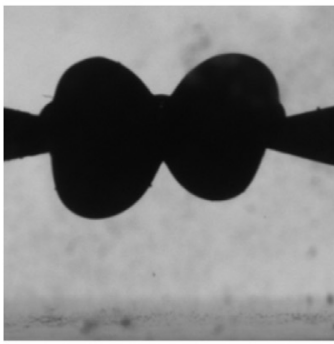

$\mathrm{t}=1$ mins

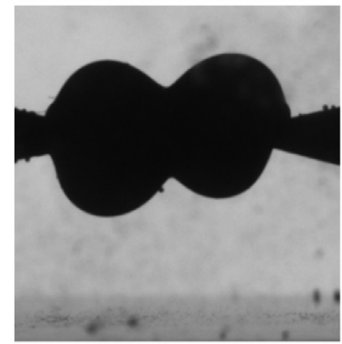

$\mathrm{t}=3$ mins

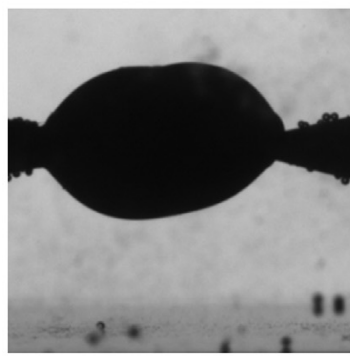

$\mathrm{t}=7$ mins

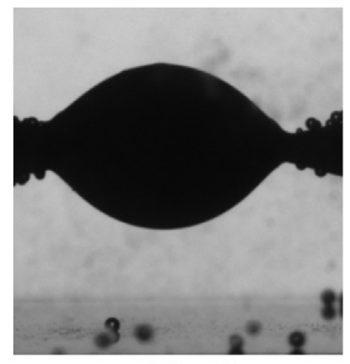

$\mathrm{t}=13$ mins

\section{Binder 50/70 [water, Temperature $50^{\circ} \mathrm{C}$ ]}

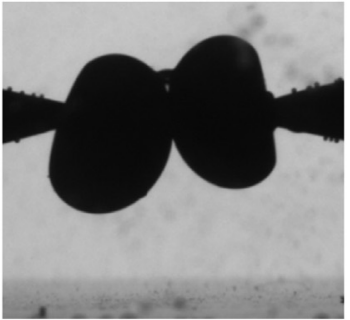

$\mathrm{t}=1 \mathrm{mins}$

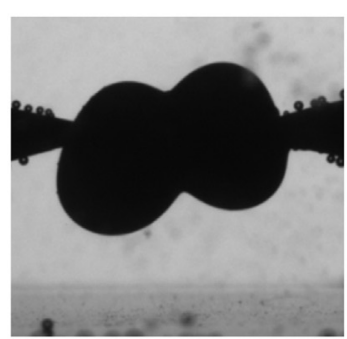

$\mathrm{t}=5 \mathrm{mins}$

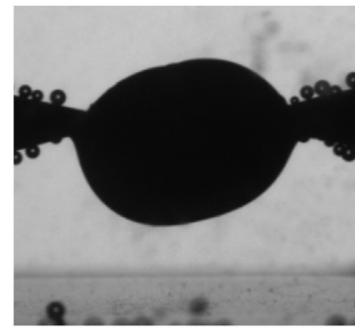

$\mathrm{t}=12 \mathrm{mins}$

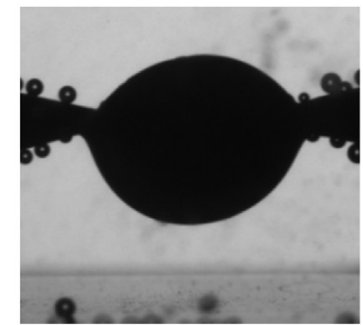

$\mathrm{t}=20 \mathrm{mins}$

Fig. 10. Coalescence of bitumen drops of three different grades in water at $50^{\circ} \mathrm{C}$.

3.5. Coalescence of bitumen drops in emulsion environment (water+emulsifier)

Emulsifiers of different types and concentrations are usually added to stabilize bitumen emulsions depending on the emulsion recipe, mineral compositions and climate conditions [47-49]. To come closer to the actual climate in which the bitumen droplets find themselves in a bitumen emulsion, the coalescence was again ana- lyzed in the same fashion as described above but adding Redicote EM44, a cationic emulsifier from AkzoNobel to the water by $1.0 \% \mathrm{wt}$. At a temperature of the water phase of $50 \mathrm{C}$ the drop relaxation times were recorded for each binder. Fig. 12 shows the representative results of the bitumen penetration grade $160 / 220,70 / 100$, and $50 / 70$.

From the analyses of the experiments, it was found that the relaxation times for each bitumen grade were enhanced 

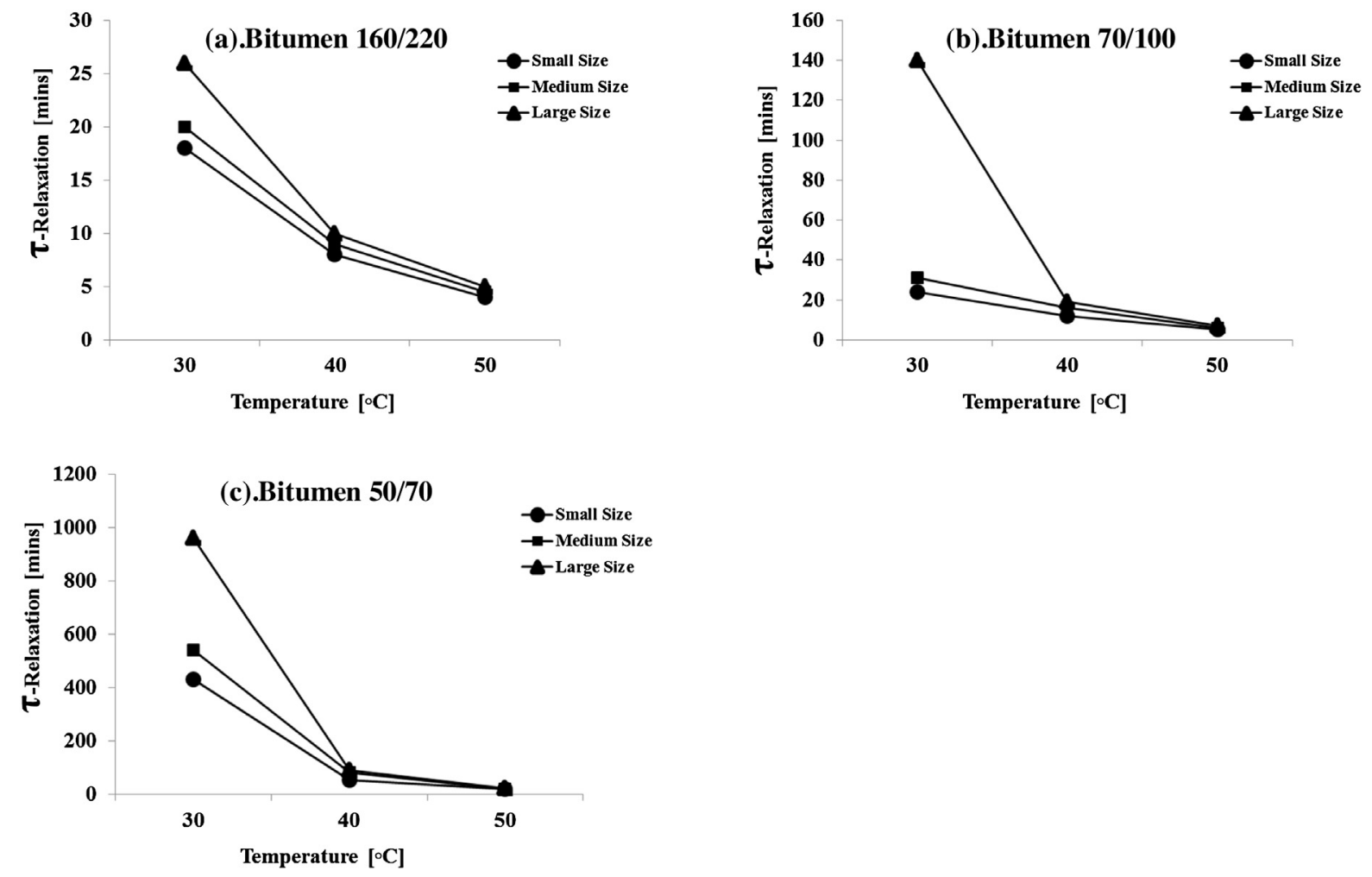

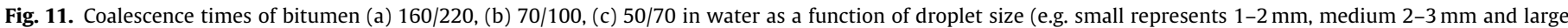
3-4 $\mathrm{mm}$ ) and temperature.

Binder 160/220 [water + Redicote EM44, Temperature $50{ }^{\circ} \mathrm{C}$ ]

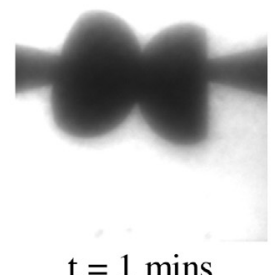

$\mathrm{t}=1$ mins

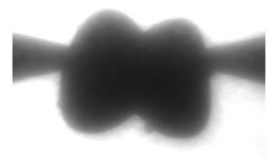

$\mathrm{t}=9$ mins

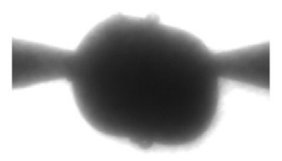

$\mathrm{t}=17 \operatorname{mins}$

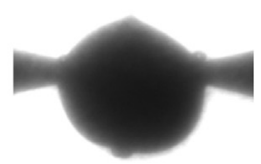

$\mathrm{t}=28 \operatorname{mins}$

Binder 70/100 [water + Redicote EM44, Temperature $50^{\circ} \mathrm{C}$ ]

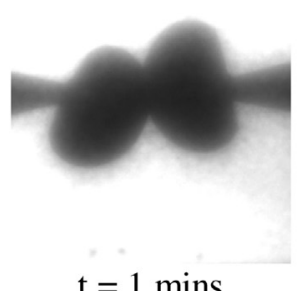

$\mathrm{t}=1$ mins

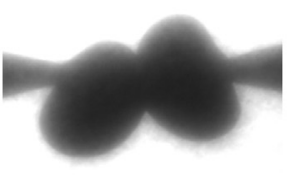

$\mathrm{t}=9$ mins

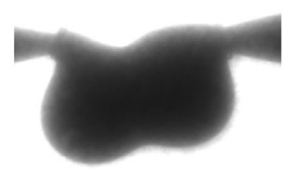

$\mathrm{t}=27 \mathrm{mins}$

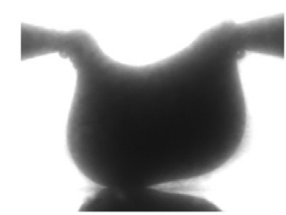

$\mathrm{t}=45 \operatorname{mins}$

Binder 50/70 [water + Redicote EM44, Temperature $50{ }^{\circ} \mathrm{C}$ ]

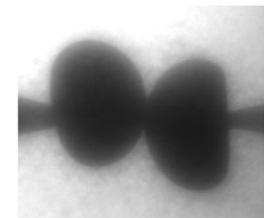

$\mathrm{t}=1$ mins

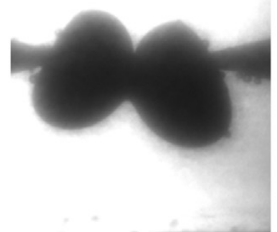

$\mathrm{t}=12 \operatorname{mins}$

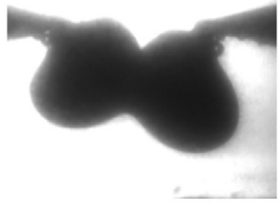

$\mathrm{t}=35 \operatorname{mins}$

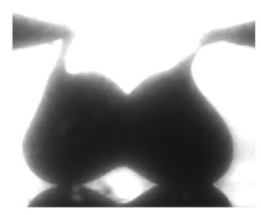

$\mathrm{t}=50 \mathrm{mins}$

Fig. 12. Coalescence of bitumen drops of three different grades in water + Emulsifier at $50^{\circ} \mathrm{C}$. 
Binder 160/220 [water + Redicote EM44@ pH 2.1, Temperature $40^{\circ} \mathrm{C}$ ]

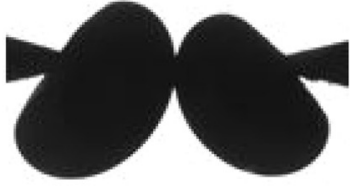

$t=0$

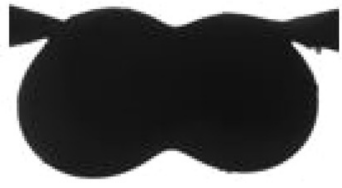

$\mathrm{t}=15 \mathrm{mins}$

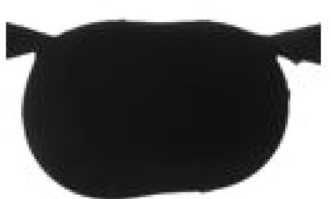

$\mathrm{t}=30 \mathrm{mins}$

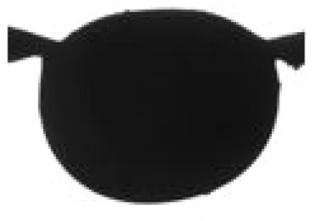

$\mathrm{t}=45$ mins

Binder 70/100 [water + Redicote EM44@ pH 2.1, Temperature $40^{\circ} \mathrm{C}$ ]

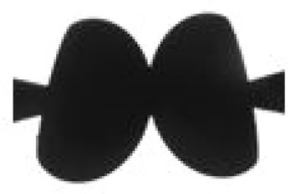

$t=0$

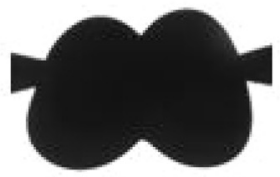

$\mathrm{t}=23 \mathrm{mins}$

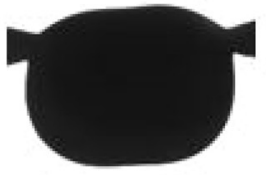

$\mathrm{t}=60 \mathrm{mins}$

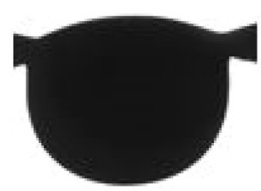

$\mathrm{t}=90 \mathrm{mins}$

Binder 50/70 [water + Redicote EM44@ @H 2.1, Temperature $40^{\circ} \mathrm{C}$ ]

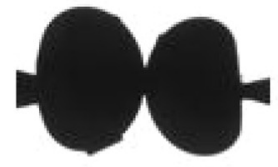

$t=0$

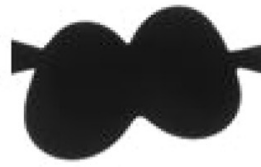

$\mathrm{t}=1 \mathrm{hr}$

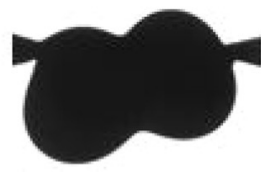

$\mathrm{t}=2 \mathrm{hrs}$

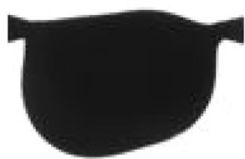

$\mathrm{t}=6 \mathrm{hrs}$

Fig. 13. Coalescence of bitumen drops of three different grades in water + Emulsifier $+\mathrm{pH} 2.1$ (hydrochloric acid) at $40^{\circ} \mathrm{C}$.

Table 4

Physic-chemical properties of water mixtures [53,54].

\begin{tabular}{|c|c|c|c|c|c|c|c|c|c|}
\hline \multirow[t]{2}{*}{ Aqueous mixtures } & \multicolumn{3}{|c|}{ Surface tension $\gamma(\mathrm{mN} / \mathrm{m})$} & \multicolumn{3}{|c|}{ Density $\rho\left(\mathrm{g} / \mathrm{cm}^{3}\right)$} & \multicolumn{3}{|c|}{ Viscosity $\eta(\mathrm{mP})$} \\
\hline & $25^{\circ} \mathrm{C}$ & $40^{\circ} \mathrm{C}$ & $50^{\circ} \mathrm{C}$ & $25^{\circ} \mathrm{C}$ & $40^{\circ} \mathrm{C}$ & $50^{\circ} \mathrm{C}$ & $25^{\circ} \mathrm{C}$ & $40^{\circ} \mathrm{C}$ & $50^{\circ} \mathrm{C}$ \\
\hline Water & 72.01 & 69.52 & 67.92 & 0.9971 & 0.9923 & 0.9881 & 8.95 & 6.54 & 5.49 \\
\hline 10\% Methanol & 56.18 & 54.01 & 52.46 & 0.9802 & 0.9747 & 0.9700 & 11.55 & 8.00 & 6.56 \\
\hline Methanol & 22.51 & 21.13 & 20.21 & 0.7869 & 0.7723 & 0.7627 & 5.41 & 4.50 & 3.96 \\
\hline $10 \%$ Ethanol & 47.53 & 45.58 & 44.26 & 0.9823 & 0.9763 & 0.9719 & 11.703 & 10.857 & 6.174 \\
\hline Ethanol & 21.82 & 20.62 & 19.82 & 0.7858 & 0.7718 & 0.7605 & 10.995 & 8.661 & 7.126 \\
\hline 10\% 2-Propanol & 40.42 & 38.43 & 37.04 & 0.9824 & 0.9761 & 0.9709 & 13.47 & 9.02 & 7.26 \\
\hline 2-Propanol & 21.22 & 19.71 & 18.69 & 0.7995 & 0.7873 & 0.7790 & 19.38 & 13.79 & 11.10 \\
\hline $10 \%$ Glycerol & 70.7 & 69.4 & 67.7 & 1.0237 & 1.0155 & 1.0113 & 11.49 & 8.26 & 6.8 \\
\hline Glycerol & 63.4 & 62.2 & 61.6 & 1.262 & 1.248 & 1.23 & 9.42 & 6.56 & 5.494 \\
\hline
\end{tabular}

due to the emulsifier additions: which were now measured as 28 min ( 4 times ${ }^{*} \tau_{\text {relaxation }}$ without emulsifier), $45 \mathrm{~min}$ (3.5 times $* \tau_{\text {relaxation }}$ without emulsifier) and $50 \mathrm{~min}$ (2.5 times $*$ $\tau_{\text {relaxation }}$ without emulsifier) for bitumen penetration grades $160 / 220,70 / 100$, and 50/70 respectively. Moreover, it can also be observed that hard binder 50/70 did not coalesce completely and instead of cohesion, adhesion happened due to sedimentation.

By comparing the effect of temperature on relaxation process, Figs. 12 and 13 provide better pictures, where relaxation time is longer in case of lower temperature of the water phase.

The presence of surfactants or surface active molecules at the interface also changes the surface tension and their variation in concentration give rise to surface tension gradients. Moreover, the contraction or expansion of the interface may also affect the concentration of surfactants, which lead to varying surface tension. In the early stage of coalescence, during neck opening, droplet interfaces contract and if emulsifiers are present then such interface shrinkage will increase their concentration, which will result in reducing surface tension. However, high concentration of surfac- 
Binder 160/220 [water + Redicote EM44, 0.5M CaCl2@ pH 2.1, Temperature 50 $\mathrm{C}$ ]

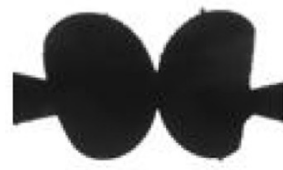

$t=0$

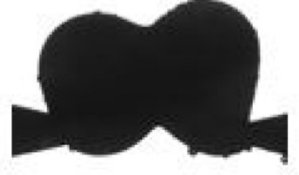

$t=10 \operatorname{mins}$

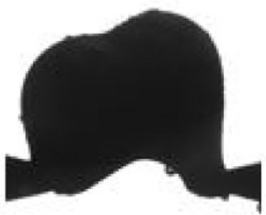

$t=15 \operatorname{mins}$

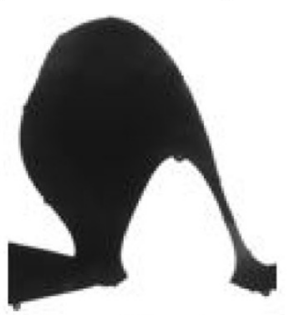

$\mathrm{t}=20 \mathrm{mins}$

Binder 160/220 [water + Redicote EM44, 0.1M CaCl @ pH 2.1, Temperature $40^{\circ} \mathrm{C}$ ]

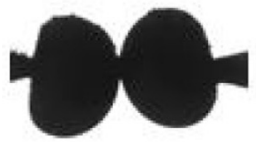

$t=0$

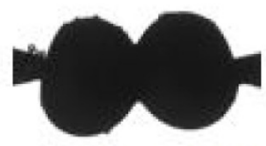

$\mathrm{t}=10 \mathrm{mins}$

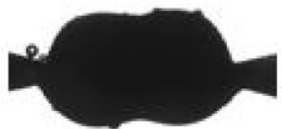

$t=30 \operatorname{mins}$

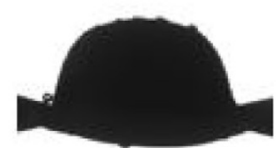

$t=60 \operatorname{mins}$

Binder 160/220 [water + Redicote EM44, 0.5M NaCl @ pH 2.1, Temperature $40^{\circ} \mathrm{C}$ ]

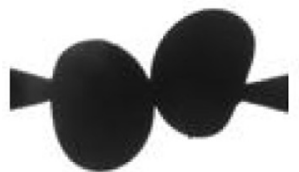

$\mathbf{t}=\mathbf{0}$

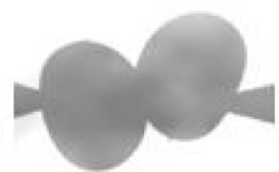

$t=10 \mathrm{mins}$

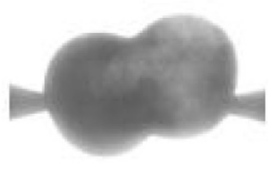

$t=30 \operatorname{mins}$

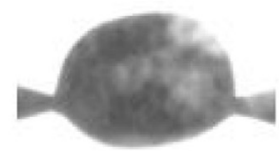

$\mathrm{t}=65 \mathrm{mins}$

Binder 70/100 [water + Redicote EM44, 0.1M CaCl @ pH 2.1, Temperature $40^{\circ} \mathrm{C}$ ]

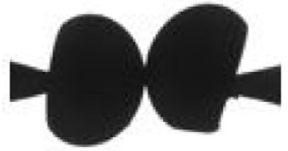

$\mathbf{t}=\mathbf{0}$

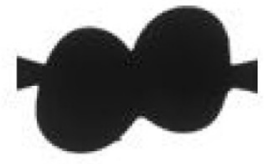

$\mathbf{t}=30 \operatorname{mins}$

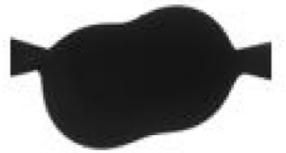

$t=90 \operatorname{mins}$

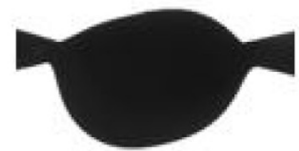

$t=140 \operatorname{mins}$

Binder 70/100 [water + Redicote EM44, 0.5M NaCl@ pH 2.1, Temperature 40 C]

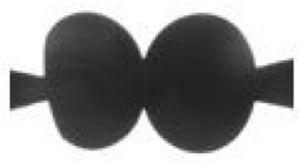

$\mathbf{t}=\mathbf{0}$

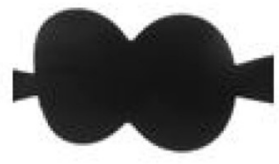

$t=30 \mathrm{mins}$

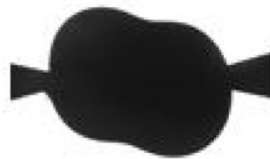

$\mathrm{t}=90 \operatorname{mins}$

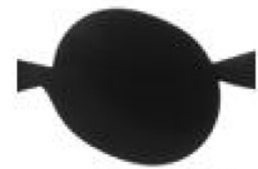

$t=170$ mins

Fig. 14. Coalescence of two bitumen drops of two different grades in water + Emulsifier + salt + pH 2.1 (hydrochloric acid) at different temperatures.

tant usually above critical micelle concentration (CMC) and a small variation in surface area will not change the surface tension.

\subsection{Coalescence of bitumen drops in emulsion environment (water + emulsifier + salt)}

This study was further prolonged to analyze the effects of salts addition to the water phase and in this regard, relaxation times were observed for the bitumen droplets without salt (see Fig. 13) and with salt additions (see Fig. 14). It was noticed that $0.1 \mathrm{M}$ addition of anhydrous $\mathrm{CaCl}_{2}$ to water phase, enhanced the coalescence time. Salt addition to bitumen water phase especially calcium chloride is very common practice. It helps to reduce the osmosis of water into the bitumen and increase the viscosity during storage. Moreover, calcium chloride also reduces the settlement of emul- 


\section{Bitumen Grade 160/220}

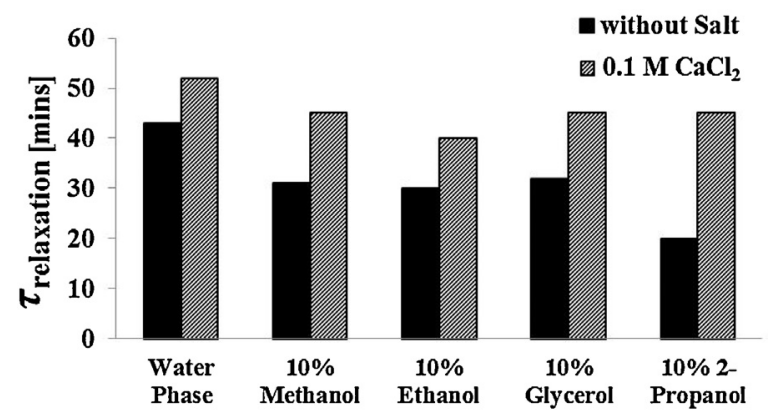

Solvents Mixed With Water Phase

Fig. 15. Coalescence of bitumen $160 / 220$ in mixed slovents with and without salt i.e. [water $+10 \%$ solvent (methanol, ethanol, 2 -propanol or glycerol) Redicote EM44, $0.1 \mathrm{M} \mathrm{CaCl}_{2} @ \mathrm{pH} 2.1$, temperature $\left.40^{\circ} \mathrm{C}\right]$.

sions by increasing the water density e.g. with $0.5 \mathrm{M}$ concentration of $\mathrm{CaCl}_{2}$.

At various salt (concentrations \& ionization potential), different phase separation situations were observed e.g. increase in water phase density at high salt concentration and phase inversion at critical salt concentration, which are consistent with the reported accelerated coalescence due to increase in salinity in the emulsions systems [50]. Moreover, the DLVO theory [51] explains the destabilizing effect of added neutral salts in emulsions which compress the double layer and reduce the repulsion potential, resulting in flocculation. Moreover, Schulze-Hardy rule state that, the ratio of critical flocculation concentrations for monovalent, divalent and trivalent ions is 100:1.6:0.13. Thus destabilization of emulsions is faster for divalent ions which are over 50 times more efficient than monovalent ions [52].

\subsection{Coalescence of bitumen drops in aqueous mixtures}

Adding other organic solvents to the water phase do not only affect the densities but also change the viscosities as well as the surface energies of the mixture phases; the main driving forces of coalescence as illustrated in Table 4.

Aqueous mixtures not only highlight the effects of surface tension variations along with minimizing the density but also ensure an inertial dominated regime for the coalescence process which is better for the stability of early neck formation process. Initially, pure water phase with and without salt was prepared for coalescence experiments but later on water was mixed with $10 \%$ methanol, ethanol, glycerol and 2-propanol. For quick analysis, softer grade bitumen (160/220) was selected as it coalesces faster than hard ones.

Fig. 15 describes the relaxation or coalescence time of bitumen droplets in mixed solvent phase with and without salt. It can be observed that presence of salt in mixed solvent environment prolonged the relaxation process.

\section{Conclusions}

This paper focused on the development and presentation of an experimental method that should enable to study the breaking of bitumen emulsions and understanding coalescence mechanisms during phase separation or water push out from bitumen emulsions. A good control on both emulsion stability and coalescence are required to improve the quality of bitumen emulsions. In the method, shape relaxation of two bitumen droplets can be experimentally tested by setting a horizontal contact between them by using L-shaped probes fixed with mechanical support while monitoring the relaxation process using a light source and a camera.

From the experiments it was shown that the relaxation times are strongly dependent on the physio-chemical properties of the bitumen and it enables a systematic investigation of the relevant parameters. From the test, the time of coalescence or relaxation ( $\left.\tau_{\text {relaxation }}\right)$ could be related to the viscosity, size of the droplet, temperature and salts addition. The initial droplet shrinkage for all three binders that were investigated was rapid as compared to the later stages of the coalescence process which confirms the expected behavior.

The following conclusions were drawn:

- It was shown that the observed Buoyancy effects on bitumen droplets in water environment could be overcome by using the horizontal test setup.

- The test was able to capture the fact that: (i) softer grade binders coalesce faster as compared to harder grade and (ii) relaxation times can be approximately compared by calculating the ratios between surface energy and viscosity $(\Upsilon / \eta)$ of binders. From the measurement of the relaxation times it was found that, at high temperatures and softer binder grades, $\tau_{\text {relaxation }}$ does not vary much with respect to droplet size. However, at lower temperatures and harder grades bitumen, influence of droplet size on $\tau_{\text {relaxation }}$ was very dominant. It was also observed that coalescence time is prolonged with large size droplets. Relaxation times for each bitumen grade were enhanced with the presence of emulsifier in the water phase.

- $0.1 \mathrm{M} \mathrm{CaCl}_{2}$ addition in an aqueous mixture inhibits the coalescence process and the relaxation times of bitumen droplets were longer as compared to water phases without salts present.

From the above the conclusion can be drawn that the developed test enables a systematic investigation of the coalescence process of two bitumen droplets, while exposed to various environments. The found relationships were logical and in line with other research performed previously. However, the advent of the test for investigating the coalescence process within bitumen emulsions relies on the assumption that a logical relationship exists between the larger scale of the test and the smaller scale of the actual emulsion droplets. This is an important aspect of the work that needs further validation. There is no need for a linear relationship between the scales, but it is important to be able to determine the scaling function. Even more important, the qualitative effect of the investigated parameters needs to be further confirmed. The authors have developed a computational approach that relies on Navier Stokes simulations and that incorporates the surface free energy behavior. As such as validation routine will be coupled to the experimental method. In the current paper however, this numerical model is not presented as the focus was placed on the test consideration itself, given the scale of research. The ability to investigate the coalescence process in a systematic way for a temperature dependent visco-elastic material may by itself already be of interest, even outside the scope of bitumen emulsions. As such, the paper by itself can have value for the research community.

A further recommendation, the test here was only demonstrated for one type and concentration of emulsifier. To further investigate the applicability of this test, many of its parameters will be varied in the continuation of this work, such as for different types of emulsifiers as well as bitumen from different crude oils and the coalescence process in the presence of a mineral substrate. Furthermore, the test results together with the numerical model, will enable the detailed development and control of bitumen emulsions which will contribute to its widespread applicability in cold mix asphalt. 


\section{Acknowledgements}

This research work was sponsored by Nynas $A B$ and performed at KTH Royal Institute of Technology, Sweden. The authors acknowledge Romain Balieu (KTH), Xiaohu Lu (Nynas), Hilde Soenen (Nynas) and Måns Collin for valuable discussions and comments.

\section{References}

[1] J. Read, D. Whiteoak, The Shell Bitumen Handbook, fifth ed., Thomas Telford Publishers, London, 2003.

[2] P. Redelius, J. Walter, Chapter 11-Bitumen emulsions, in: J. Sjöblom (Ed.), Emulsions and Emulsions Stability, second ed., CRC Press Taylor \& Francis Group, 2005, pp. 384-412.

[3] D.H. Melik, H.S. Folger, Turbidimetric determination of particle size distributions of colloidal systems, J. Colloid Interface Sci. 92 (1983) 161-180.

[4] J. Gregory, Turbidity fluctuation in flowing suspensions, J. Colloid Interface Sci. 105 (1985) 357-371.

[5] J. Eisenlauer, D. Horn, Fibre-optic sensor technique for flocculant dose control in flowing suspensions, Colloids Surf. 14 (1985) 121-134.

[6] H.H. Trimm, B.R. Jennings, K. Parslow, Colloid deflocculation detection by transient light scattering, Colloids Surf. 18 (1986) 113-121.

[7] A. Menchaca-Rocha, A. Martinez-Davalos, R. Nunez, Coalescence of liquid drops by surface tension, Phys. Rev. E 63 (2001), 046309-1-5.

[8] Z. Mohamed-Kassim, E.K. Longmire, Drop coalescence through a liquid/liquid interface, Phys. Fluids 16 (2004) 2170-2181.

[9] P.J. Graham, M.H. Farhangi, A. Dolatabadi, Dynamics of droplet coalescence in response to increasing hydrophobicity, Phys. Fluids 24 (2012), 112105-1-20.

[10] D.J. Miller, Coalescence in crude oil emulsions investigated by a light transmission method, J. Colloid Polym. Sci. 265 (1987) 342-346.

[11] E. Evans, D. Needham, Physical properties of surfactant bilayers membranes: thermal transitions, elasticity, cohesion, and colloidal interactions, J. Phys. Chem. 91 (1987) 4219-4228.

[12] A. Yeung, T. Dabros, J. Masliyah, J. Czarnecki, Micropipette: a new technique in emulsion research, Colloids Surf. A: Physicochem. Eng. Aspects 174 (2000) $169-181$.

[13] J.V. Kloet, L.L. Schramm, B. Shelfantook, The influence of bituminous froth components on water-in-oil emulsion stability as determined by the micropipette technique, Colloids Surf. A: Physicochem. Eng. Aspects 192 (2001) 15-24.

[14] J.W.S. Rayleigh, Further observations upon liquid jets, Proc. R. Soc. Lond. 34 (1879) 130-145.

[15] O. Reynolds, On the floating of drops on the surface of water depending only on the purity of the surface, Proc. Manchester Lit. Phil. Soc. 21 (1881).

[16] J. Eggers, Coalescence of spheres by surface diffusion, Phys. Rev. Lett. 80 (1998) 2634-2637

[17] R.W. Hopper, Plane Stokes flow driven by capillarity on a free surface, J. Fluid Mech. 213 (1990) 349-375.

[18] R.W. Hopper, Stokes flow of a cylinder and half space driven by capillarity, J. Fluid Mech. 243 (1992) 171-181.

[19] R.W. Hopper, Coalescence of two viscous cylinders by capillarity: part-I-theory part-II-shape evolution, J. Am. Ceram. Soc. 76 (1993) 2947-2960.

[20] J.I. Martinez-Herera, J. Derby, Viscous sintering of spherical particles via finite element analysis, J. Am. Ceram. Soc. 78 (1995) 645-649.

[21] J.D. Paulsen, J.C. Burton, S.R. Nagel, S. Appathurai, M.T. Harris, O.A. Basaran, The inexorable resistance of inertia determines the initial regime of drop coalescence, Proc. Natl. Acad. Sci. 109 (2012) 6857-6861.

[22] F. Blanchette, L. Messio, J.W.M. Bush, The influence of surface tension gradients on drop coalescence, Phys. Fluids 21 (2009), 072107-1-10.

[23] M. Wu, T. Cubaud, C.M. Ho, Scaling law in liquid drop coalescence driven by surface tension, Phys. Fluids 16 (2004) L51-54.

[24] H.A. Stone, A.D. Stroock, A. Ajdari, Engineering flows in small devices: microfluidics toward a lab-on-a-chip, Annu. Rev. Fluid Mech. 36 (2004) $381-411$

[25] J. Atencia, D.J. Beebe, Controlled microfluidic interfaces, Nature 437 (2005) 648-655.
[26] W.D. Ristenpart, P.M. McCalla, R.V. Roy, H.A. Stone, Coalescence of spreading droplets on a wettable substrate, Phys. Rev. Lett. 97 (2006) 064501-1-4.

[27] L.Y. Yeo, R.V. Craster, O.K. Matar, Drop manipulation and surgery using electric fields, J. Colloid Interface Sci. 306 (2007) 368-378

[28] K. Olsson, R. Lundberg, M. Wiklund, U. Lillbroända, P. Redelius, New Cold Paving Technology, Recycling and Virgin Mixes, CME, Lyon, France, 2002.

[29] D. Lesueur, J.J. Potti, Cold mix design: a rational approach based on the current understanding of the breaking of bituminous emulsions, in: 3rd Eurasphalt and Eurbitumen Congress, Vienna, Austria, May, 2004, pp. 407-421.

[30] European Standard EN 13075-1, Bitumen and bituminous binders-Determination of breaking behaviour-Part 1: Determination of breaking value of cationic bitumen emulsions, mineral filler method.

[31] C. Such, G. Ramond, F. Durrieu, P. Zannis, J.P. Antoine, M. Ullman, P. Bothorel, Caractérisation des émulsions de bitume pour enduits superficiel, Bull. Liason. Labo B. et Ch. 169 (September-October) (1990), Ref 3501

[32] J.L. Marchal, Evaporation-filtration test for emulsion inversion, Asphalt Emulsions, ASTM STP 1079 (1990) 106-111.

[33] J. Walter, D. Day, Coalescence of Quick Set Surface Dressing PMB Emulsions, CME, Lyon, France, 2002.

[34] A. Banerjee, A. Bhashin, J. Prozzi, Characterizing stability of asphalt emulsions using electrokinetic techniques, J. Mater. Civil Eng. 25 (2013) 78-85.

[35] M. Bourrel, E.F. Verzaro, Mécanismes de rupture des émulsions de bitume routier, J. Acta Chim. 2-3 (1996) 42-48.

[36] J.L. Keddie, Film formation of latex, J. Mat. Sci. Eng. 21 (1997) 101-170.

[37] L. Bonakdar, J. Philip, P. Bardusco, J. Petkov, J.J. Potti, P. Meleard, F. Leal-Calderon, Rupturing of bitumen-in-water emulsions: experimental evidence for viscous sintering phenomena, Colloid Surf. A: Physiochem. Eng. Aspects 176 (2001) 185-194.

[38] W. Kang, L. Guo, H. Fan, L. Meng, Y. Li, Flocculation, coalescence and migration of dispersed phase droplets and oil-water separation in heavy oil emulsions, J. Pertoleum Sci. Eng. 81 (2012) 177-181.

[39] J. Frenkel, Viscous flow of crystalline bodies under the action of surface tension, J. Phys. (Mosc. USSR) 9 (1945) 385-391.

[40] G.A.L.D. Aarts, H.N.W. Lekkerkerker, H. Guo, G.H. Wegdam, D. Bonn, Hydrodynamics of droplet coalescence, Phy. Rev. Lett. 95 (2005), 164503-1-4.

[41] P. Becher Edition, Encyclopedia of Emulsion Technology, vol. 1, Marcel Dekker Inc., New York, USA, 1983.

[42] P. Becher Edition, Encyclopedia of Emulsion Technology, vol. 2, Marcel Dekker Inc., New York, USA, 1985

[43] S.E. Friberg, Emulsion stability, in: J. Sjöblom (Ed.), Emulsions-A Fundamenta and Practicle Approach, Kluwer Academic, Springer Science, 1992, pp. 1-24.

[44] R.A. Mohammed, A.I. Bailey, P.F. Luckham, S.E. Taylor, Dewatering of crude oil emulsions 1, Colloids Surf. A: Physicochem. Eng. Aspects 80 (1993) 223-235.

[45] S. Dukhin, O. Saether, J. Sjöblom, Encyclopedic Handbool of Emulsion Technology, in: J. Sjöblom (Ed.), Marcel Dekker Inc., New York, USA, 2001, pp. 71-93.

[46] A. Khan, P. Redelius, N. Kringos, Toward understanding breaking and coalescence of bitumen emulsions for cold asphalts, in: Paper 402 (Accepted Manuscript in EE Proceedings), 6th Euroasphalt \& Eurobitume Congress, Czech Republic, June 1-3, 2016.

[47] R. Jean-Claude, Routes de France, Section SFERB, Bitumen Emulsions, Paris, 2008.

[48] Akzo Nobel, Bitumen emulsion Technical Bulletin, 03294-300410, http://sc. akzonobel.com/en/asphalt/Documents/AN_Asphalt_Emulsion_TB_eng.pdf.

[49] E.A. Kamba, A.U. Itodo, E. Ogah, Utilization of different emulsifying agents in the preparation and stabilization of emulsions, Int. J. Mater. Chem. 3 (2013) 69-74.

[50] Q. Yang, D. Ke, M. Yang, J. Hong, Q. Ran, X. Wang, Effect of salt concentration on the phase separation of bitumen emulsions, Colloid Surf. A: Physiochem. Eng. Aspects 425 (2013) 1-5.

[51] Advanced Dairy Chemistry Volume 2 Lipids, in: P.F. Fox, P.L.H. McSweeney (Eds.), 3rd ed., Springer, New York, USA, 2006.

[52] S.E. Friberg, Emulsion stability, in: S.E. Friberg, K. Larsson (Eds.), Food Emulsions, 3rd ed., Marcel Dekker Inc., New York, USA, 1997, pp. 1-55.

[53] S.Z. Mikhail, W.R. Kimel, Densities and viscosities of methanol-water mixtures, J. Chem. Eng. Data 6 (1961) 533-537.

[54] G. Vázquez, E. Alvarez, J.M. Navaza, Surface tension of alcohol + water from 20 to $50^{\circ} \mathrm{C}$, J. Chem. Eng. Data 40 (1995) 611-614. 